\title{
Multi-agent Simulation of NAS Infrastructure Utilization in the Presence of Airline Policies
}

\author{
Guillermo Calderón-Meza (Ph.D. Candidate), CATSR - George Mason University, Fairfax, VA \\ Lance Sherry (Ph.D), CATSR - George Mason University, Fairfax, VA \\ María Consiglio (Ph.D), NASA, Hampton Roads, VA
}

\section{Abstract}

\section{Introduction}

The symptoms of saturation that the Air Transportation System of the United States (NAS) shows $\left.{ }^{1}\right]$ have motivated the proposal of investment to increase capacity. Previous research shows that adaption of the stakeholders could lead to under-utilization of the infrastructure. This and the cost of the new infrastructure motivate studies that can predict how well infrastructure will be used.

Airlines have the goal of obtaining profit while other stakeholders have goals like reducing delay and traffic balancing. When stakeholders try to achieve their own goals (assuming "rational agents" $\left[{ }^{2}\right]$ ) the situation becomes a Nash Equilibrium as defined by Game Theory: none of the agents voluntarily change their strategy without incentives. However, some games can perform better than the Nash Equilibrium if the agents share information $\left[{ }^{3},{ }^{4}, 5\right]$. Proposed NextGen technologies such as the System Wide Information Management (SWIM) will provide more real-time information to the stakeholders. With this information agents will use alternative strategies or other forms of interaction $\left[^{6}\right]$ affecting both performance and utilization.

Creating tools to evaluate the potential effects of the changes to the NAS is an important research goal $\left[{ }^{7},{ }^{8},{ }^{9}\right]$. This paper uses a multi-agent-based simulation to model aircraft operations and infrastructure utilization in the presence of airline policies and aircraft route selection strategies. The main hypothesis of the paper is that strategies other than the current first come-first served (FCFS) can improve the utilization of the system, keep the cost low, and give more flexibility to the airlines and individual flights.

\section{Problem description}

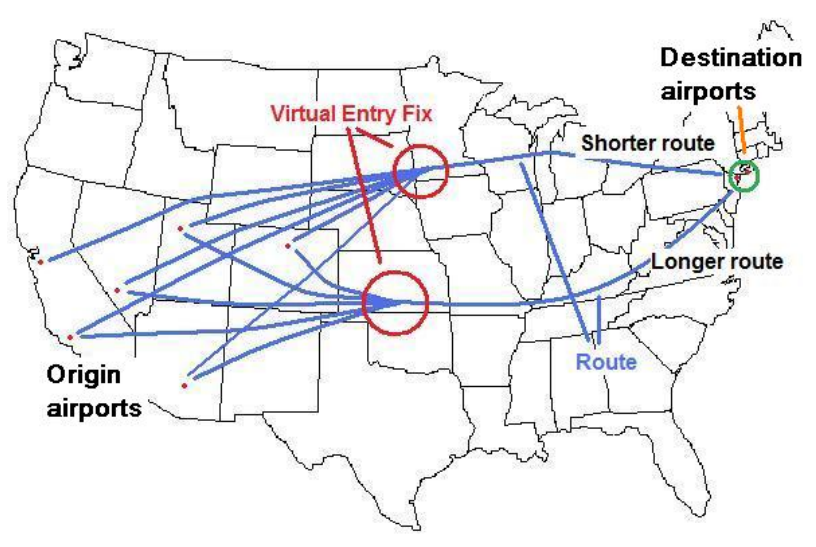

Figure 1: Conceptual representation of the situation being modeled

Aircraft scheduled to depart from several airports on one side of the country can choose from a set of alternate trans-continental routes to their common destination airport (or metroplex) on the other side of the country (see Figure 1). Controllers assign flights to routes so that the traffic is balanced among routes, in a first come-first served discipline. The routes are divided into slots to model the safety rules (separation distances). Usually, aircraft wait on the ground until the controllers allow them to fly just-in-time to enter an available slot in a route. Delays on the ground imply wait times and costs for passengers and airlines. Delays can be modeled by departure queues. Once an aircraft enters a route, it flies at constant speed to maintain separation distances with the other aircraft flying the same route. 


\section{Methodology}

An assumption is that aircraft push back from the gates with a push back delay that is exponentially distributed. Each route has its own entry queue (or wait) associated. The queues are also divided into slots, time slots. The entry queue is virtually located before the so-called virtual entry fix which is the point in which all the aircraft enter the route (see Figure 1). The number of slots from the entry fix to the destination is called the route length. In this study, all slots are either 5 minutes (for time slots) or $25 \mathrm{~nm}$ (for distance slots). A constant speed of 300 knots is assumed so that it takes 5 minutes to fly $25 \mathrm{~nm}$.

When more resources or information are available, as it is proposed by NextGen (SEVEN, SWIM $\left[{ }^{10}\right]$ ) the airlines or the flight crews themselves could choose routes to maximize their objective functions.

The strategy of a flight agent is modeled by the following equation:

$$
i=\underset{i}{\operatorname{argmin}}\left[\phi * c_{f} * d\left(r_{i}\right)+(1-\phi) * c_{w} * q\left(r_{i}\right)\right]
$$

Where $r_{i}$ is the $i$-th route, $\varphi$ is the control variable (parameter) so that $\beta \leq \varphi \leq \alpha, d(x)$ is the distance of route $x, q(x)$ is the length of the entry queue for the route $x, c_{f}$ is the cost of flying a distance slot, $c_{w}$ is the cost of waiting a time slot.

The policy of an airline is modeled by two parameters, $\beta$ and $\alpha$. The conditions on these parameters are $0 \leq \beta<\alpha \leq 1$. Any combination of policies can be simulated by changing the value of these parameters for each airline.
A flight can use any value of $\varphi$ that the policy allows. This allows several strategies for the route selection by varying $\varphi$. Setting $\varphi=0$ obtains the first come-first served strategy, i.e. the flight decides based on the length of the waiting queue. Other examples are $\varphi=1$, i.e. the flight decides based on the route distance, and $\varphi=0.5$, i.e. give the same relevance to the length of the waiting in queue and the distance of the route. Also, setting $\beta$ $=\alpha$ forces all the flights of an airline to use the same strategy, i.e. a single point strategy.

The aircraft are rational agents $\left[{ }^{2}\right]$. The agents decide based on local information only: no systemwide, historic or future information is considered in this version of the simulator.

These experiments are simulated using the MASON discrete event multi-agent simulation platform $\left[{ }^{11}\right]^{1}$. MASON is a free, open-source, Java library designed and implemented at George Mason University.

Each airline agent in the model defines the policy for its flights. Each flight agent defines its weights, in the moment of its creation, by asking the airline what is the current policy and choosing randomly from the ranges of that policy. There are 90 flight agents because each airline has a schedule with 30 flights. Only flat schedules are used in this study: there is only one aircraft per time slot, and there are no gap in the schedules, i.e. flights are all consecutive. The task of the flight agents during the simulation is to decide which route to take. After that, route agents handle the flight agents. Route agents have several tasks. First, route agents receive new flights in the route. Second, they model the motion of the flights in the route. Third, they take out of the route flights that reach their destination, i.e. the end of the route. Fourth, each route agent contains a waiting (entry) queues and move flights in and out of the queue -and into the route-. There are other agents to gather statistics and to store data into a database for further analysis.

This study consists of eight experiments, one for each value of $\varphi$-strategy- from the set $\{0.0,0.1$, $0.2,0.33,0.5,0.67,0.75,1.0\}$. The are two routes

\footnotetext{
${ }^{1}$ See http://cs.gmu.edu/ eclab/projects/mason/ for more information.
} 
in each experiment, one of them with fixed distance of 57 slots. The other route varies its distance from 39 to 75 slots in 2 slots steps, to have 19 distance ratios in total. Three wait to fly cost ratios are also used in each experiment: $0.3,0.5$, and 0.7. So, each experiment has 57 scenarios, one for each combination of route distance and wait to fly cost ratios. Each scenario is repeated 30 times (Monte Carlo simulation) to account for the stochastic nature of the simulation. Each repetition of a scenario runs for 100 time steps.

\section{Results and discussion}

Figure 2 is the result for three airlines, two routes, and average push back delay of 25 minutes (exponentially distributed), and a wait/fly cost ratio of 0.3. In the experiment, the distance ratio varied from 0.68 to 1.32 . Also the phi parameter varied in several steps from 0 to 1 . All the values shown in the image are averages taken over 30 executions of the same scenario. One standard deviation above and below the mean value is also shown by the whiskers. The figure shows that the $\varphi$ and the route distance ratio parameters are two of the variables that control the number of unused route slots. Other variables are the cost of waiting to cost of flying ratio (wait/fly ratio), total number of scheduled flights, number of routes, and number of airlines. But of these other variables only the wait/fly ratio will be explored in this study.

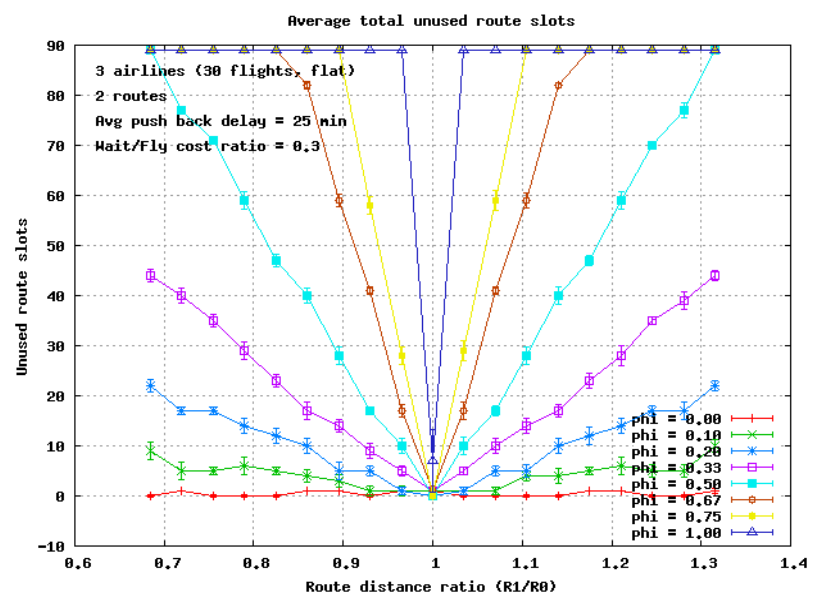

Figure 2: Average total unused route slots as function of the distance ratio and the $\varphi$ value for o woit/flv motio of $\cap 2$
The best utilization is achieved when $\varphi=0$. So, choosing routes based only on route distance is the best a flight or a controller can do if the goal is to optimize utilization only.

When the route distance ratio approaches 1 , the number of unused slots approaches 0 , but the standard deviation increases. In that point, all values of $\varphi$ make the system behave the same way, i.e. all strategies are equivalent. Strategies show their differences as the distance ratio moves away from 1. Lower values of $\varphi$ result in less unused route slots than higher values do. But the differences in the number of unused slots are not linear on $\varphi$.

Figure 3 is the result for the set of parameters used for Figure 2, but the wait/fly ratio is 0.5 instead of 0.3 . The figure shows the effect of increasing the wait/fly ratio. The $\varphi=0.00$ and $\varphi=$ 1.00 do not show any difference with respect to the results shown in Figure 2 regardless of the increment of the ratio. All the other values of $\varphi$ show a reduction in the number of unused route slots with respect to the values shown in Figure 2. The reduction in unused slots is significant, it in some cases it approaches $40 \%$. Increasing the wait/fly ratio makes the agents use the resources better.

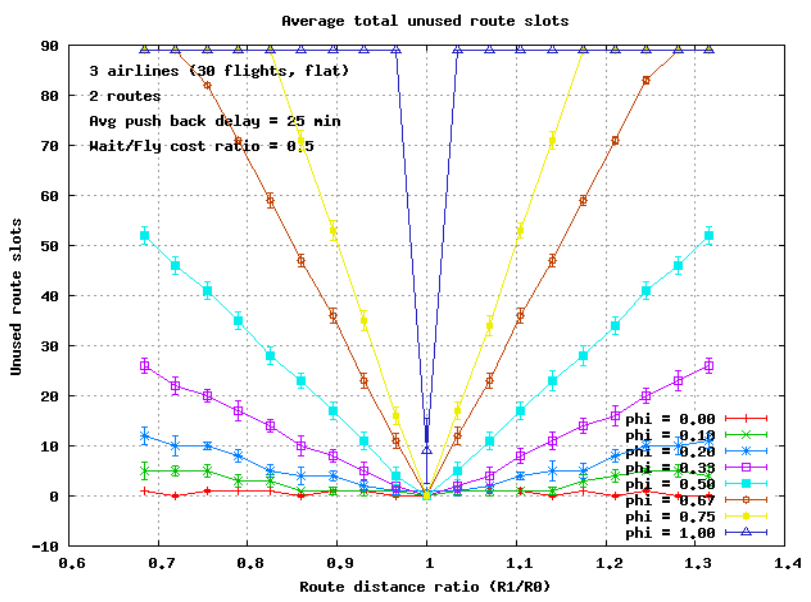

Figure 3: Average total unused route slots as function of the distance ratio and $\varphi$ value for a wait/fly ratio of 0.5

Figure 4 is the result for the set of parameters used for Figure 2 and Figure 3, but the wait/fly ratio is 0.7 instead of 0.3 or 0.5 . When $\varphi=0.00$ and $\varphi=$ 
1.00 the behavior of the system in terms of unused route slots is the same as it was in the two previous cases. But the rest of the values show reductions in the number of unused route slots even when compared to the values for a wait/fly ratio of 0.5 (Figure 3). However, the reduction is smaller than it was when the wait/fly ratio when from 0.3 to 0.5 .

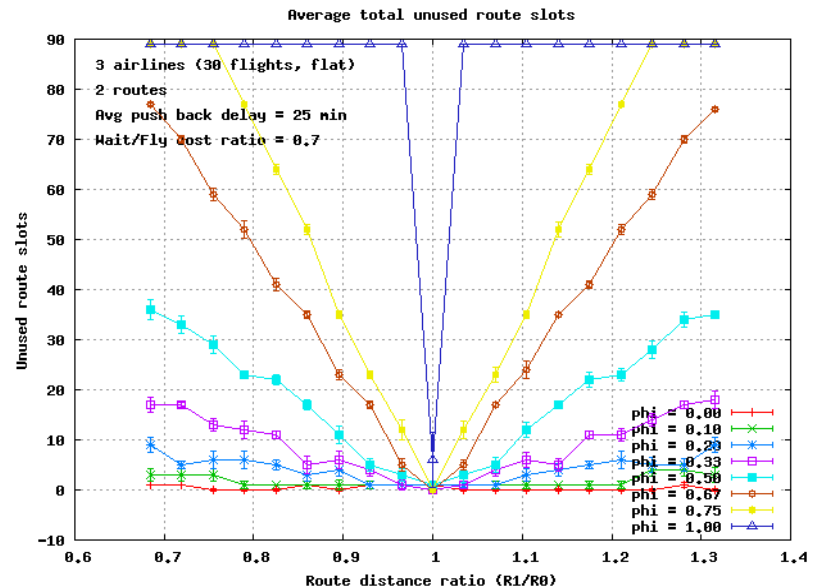

Figure 4: Average total unused route slots as function of the distance ratio and $\varphi$ value for a wait/fly ratio of 0.7

The figures above show that, in terms of route utilization, the effect of the strategies used by the flights is more evident as the distance ratio moves away from 1 . When the distance ratio is 1 , there is no difference between strategies. The best strategy is to select the shortest wait queue $(\varphi=0.0)$ regardless of any other variable or parameter. If the wait/fly ratio increases, the strategies even improve the utilization.

Figure 5 is the result that complements the utilization shown in Figure 2, and the values where obtained in the same experiment. The figure shows that the variables controlling the utilization also control the aggregated airline cost. In general, if the distance ratio is close to 1 , choosing a lower value of $\varphi$ will reduce the cost in general. When the distance ratio deviates too much from 1.0, then higher values of $\varphi$ reduce the costs better than lower values.

When the distance ratio is 1 , all the strategies behave the same way, i.e. in that case any strategy can be used.

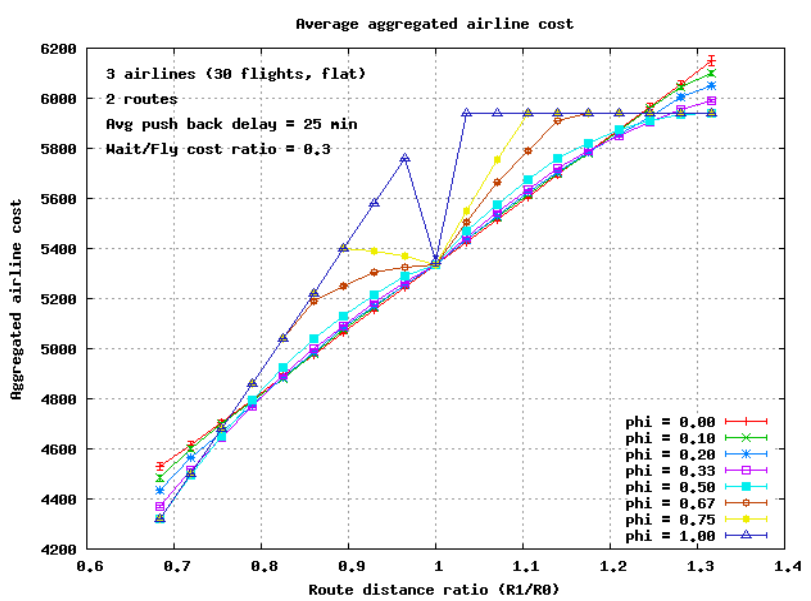

Figure 5: Average aggregated airline cost as function of the distance ratio and phi value for a wait/fly ratio of 0.3

Figure 6 shows the results that complement the utilization shown in Figure 3. The comparison between Figure 5 and Figure 6 shows that when the wait/fly ratio increases the effect of $\varphi$ is accentuated; higher values of $\varphi$ increase the aggregated airline costs with respect to lower values of $\varphi$, and the relative differences between the behaviors become bigger. It is not observed anymore in the figure if there is a crossing of the lines when the distance ratio moves away from 1 . But again, when the distance ratio is 1 , there is no difference between strategies.

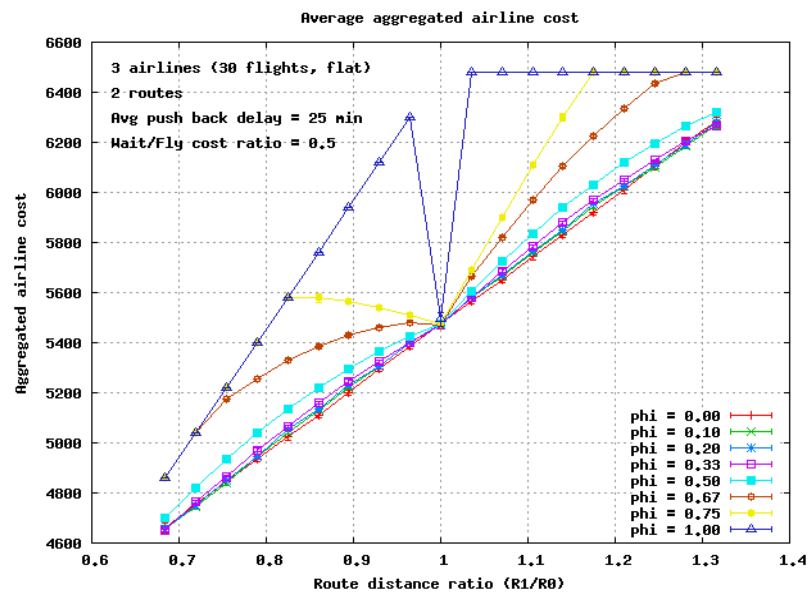

Figure 6: Average aggregated airline cost as function of the distance ratio and phi value for a wait/fly ratio of 0.5

Figure 7 shows that increasing the the wait/fly cost accentuates the difference between strategies; the lines separate even more from each other in this 
case. However, all the strategies behave the same way when the distance ratio is 1.0 .

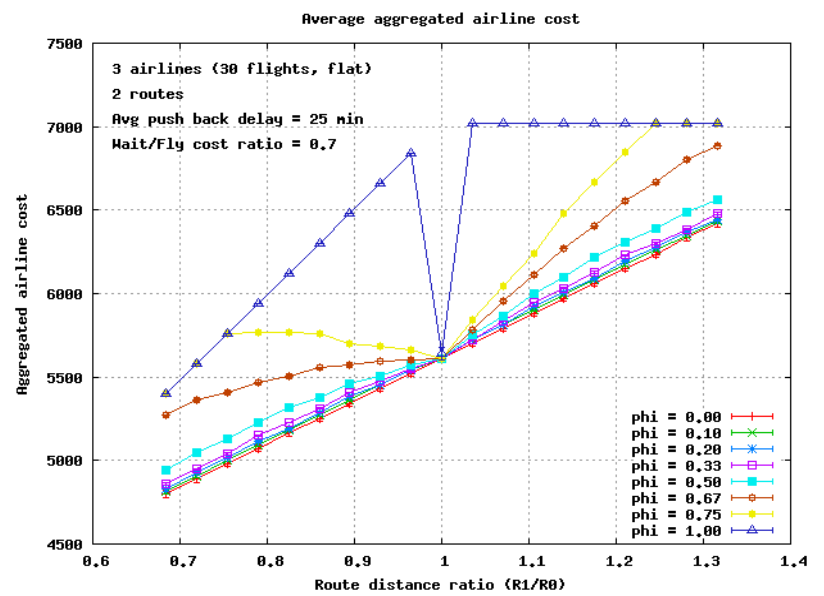

Figure 7: Average aggregated airline cost as function of the distance ratio and phi value for a wait/fly ratio of 0.7

Summarizing, from the aggregated airline cost point of view, choosing lower values of $\varphi$ reduces the cost when compared to the higher values of $\varphi$. An exception is when the wait/fly ratio is low and the distance ratio deviates too much from 1 . In this case, using higher values of $\varphi$ achieves lower aggregated costs. Increases in the wait/fly ratio only accentuate the differences between strategies, but still lower values of $\varphi$ achieve, in general, lower aggregated airline costs.

\section{Conclusions and future work}

In system where all the flights simultaneously use the same strategy, controlling the aggregated airlines cost and the route utilization of the system takes a combination of several variables: the strategy of the flights (determined by the $\varphi$ parameter), the wait/fly ratio, and the route distance ratio. The wait/fly ratio is usually given, it is a characteristic of the system. Then, in fact, only two variables can really be used to make decisions in a system like this.

From the utilization point of view, the best strategy is $\varphi=0.0$, i.e. choosing routes based on the shortest wait queue. If the distance ratio is 1.0 there is no difference between strategies.
From the point of view of aggregated airline cost, the best strategy uses lower $\varphi$ values. There is an exception when wait/fly ratio is low and distance ratio deviates too much from 1.0. In this particular case it is better to use higher values of $\varphi$ for the flights. An increase in the wait/fly ratio only accentuates the difference between strategies. But it does not change the fact that, in general, using lower values of $\varphi$ achieves lower costs than higher values of $\varphi$.

The contribution of this paper is the conclusion that FCFS strategy $(\varphi=0.0)$ is usually correct when assigning routes to flights. However, there are some exceptions to this statement. Pilots and controllers should be aware of these exceptions so that they can help achieve higher performance when these exceptional conditions take place.

Work is being done to create adaptable agents that seek improvements of global utilization and aggregated airline costs. The current implementations uses evolutionary algorithms to achieve this adaptation. With these methods, the limitation of using the same strategy for all the flights is not needed.

\section{Acknowledgments}

The authors acknowledge the help provided by Dr. Robert L. Axtell, Dr. Kenneth A. De Jong, Dr. Thomas Speller Jr., all faculty from George Mason University, during this study and related research.

\section{Disclaimer}

If a disclaimer is necessary, include it under the heading Disclaimer using the Heading 1 style. Use the Body Text/Normal Text style and include the disclaimer immediately after the references.

\section{Email Addresses}

The e-mail of Guillermo Calderón-Meza is gcaldero@gmu.edu. The e-mail of Dr. Lance Sherry is lsherry@gmu.edu. 


\section{Conference Identification}

In order to identify at which ICNS Conference the paper was published and presented; please add the following lines at the end of the paper using the Author text style:

\section{ICNS Conference}

13-15 May 2009

\section{$1 \quad$ References}

Edwards, Eric; Donohue, George L.; Shaver, Russell D. III. Terminal chaos: why US air travel is broken and how to fix it. American Institute of Aeronautics and Astronautics, Inc, USA, 2008. ISBN 978-1-56347-946-6.

${ }^{2}$ Russel, Stuart and Norvig, Peter. Artificial Intelligence. A Modern Approach. Prentice Hall, USA, 2003. ISBN 0-13-790395-2.

${ }^{3}$ Knorr, Dave; Wetherly, James; Wambsganss, Mike; Ball, Michael O.; Hofman, Robert L.

Assessing the benefits of collaborative decision making in air traffic management. Air Transportation Systems Engineering, 193:239-250, 2001. ISBN 1-56347-474-3.

${ }^{4}$ Bouge, Nicolas; Vial, Sophie; Martin, Peter; Hudgell, Alison. Improved information sharing: A step toward the realization of collaborative decision making. Air Transportation Systems Engineering, 193:2161-175, 2001. ISBN 1-56347-474-3.

${ }^{5}$ Davidson, Hayley J.; Hansmann, R. John. Effect of shared information on pilot/controller and controller/controller interactions. Air Transportation Systems Engineering, 193:205-223, 2001. ISBN 156347-474-3.

${ }^{6}$ Ferber, Jacques. Multi-Agent Systems. An Introduction to Distributed Artificial Intelligence. Addison-Wesley, United Kingdom, 1999. ISBN 0201-36048-9.

${ }^{7}$ Greenbaum, Daniel P.; Wojcik, Leonard A.; Campbell, Keith C.; Cooper, Wayne W. Jr. Modeling distributed human decision making in traffic flow management operations. Air Transportation Systems Engineering, 193:227\{237, 2001. ISBN 1-56347-474-3.
${ }^{8}$ Tomlin, Clare J.; Waslander, Steven L. Efficient market-based air traffic control flow with competing airlines. Proceedings of the 45th IEEE Conference on Decision and Control, pages 28432848, December 2006.

${ }^{9}$ Wojcik, Leonard A. Airline personalities and air traffic flow management: A simple agent-based model. In AIAA 4th Aviation Technology, Integration and Operations Forum, pages 1-16, Chicago, Illinois, USA, September 2004.

${ }^{10}$ Dieudonne, J.E.; Crane, H.L.; Gonda, J., and Jones, S.R. Neo (NextGen TBOs) provided by multi-agency surveillance SOA (SDN). Digital Avionics Systems Conference, 2007. DASC '07. IEEE/AIAA 26th, pages 1.E.3-1-1.E.3-13, Oct. 2007.

${ }^{11}$ Panait, Liviu; Sullivan, Keith; Luke, Sean, CioffiRevilla, Claudio. Mason: A new multi-agent simulation toolkit. In Proceedings of the 2004 SwarmFest Workshop, 2004. 
